\title{
Global attractor for the weakly damped forced KdV equation in Sobolev spaces of low regularity
}

\author{
Xingyu Yang
}

\begin{abstract}
We consider the global attractor for the weakly damped forced $\mathrm{KdV}$ equation in Sobolev spaces $\dot{H}^{s}(\mathbf{T})$ for $s<0$. Under the assumption that the external forcing term belongs to $\dot{L}^{2}(\mathbf{T})$, we prove the existence of the global attractor in $\dot{H}^{s}(\mathbf{T})$ for $-1 / 2 \leq s<0$, which is identical to the one in $\dot{L}^{2}(\mathbf{T})$ and thus is compact in $H^{3}(\mathbf{T})$. The argument is a combination of the I-method and decomposing the solution into two parts, one of which is uniformly bounded in $\dot{L}^{2}(\mathbf{T})$ and the other decays exponentially in $\dot{H}^{s}(\mathbf{T})$.
\end{abstract}

Mathematics Subject Classification (2010). 35Q53, 37L50.

Keywords. KdV, Global attractor, Asymptotic smoothing effect, I-method.

\section{Introduction and the main results}

We consider the global attractor for the weakly damped forced KdV equation

$$
\partial_{t} u+\partial_{x}^{3} u+\frac{1}{2} \partial_{x} u^{2}+\gamma u=f, \quad x \in \mathbf{T}, \quad t>0,
$$

in $\dot{H}^{s}(\mathbf{T})$ for $s<0$, where the unknown $u$ is real-valued, and $\gamma>0$ is a real constant, and the forcing term $f \in \dot{L}^{2}(\mathbf{T})$ is time independent, and $\mathbf{T}$ is the one-dimensional torus, and $\dot{H}^{s}(\mathbf{T})=\left\{u \in H^{s}(\mathbf{T}): \int_{\mathbf{T}} u d x=0\right\}$.

For $\gamma=0$ and $f=0$, Eq. (1.1) is the well-known $\mathrm{KdV}$ equation, which was first derived by Korteweg and de Vries [1] to model water waves in a shallow canal, and then was used to describe approximately the evolution of one-dimensional long waves in many physical settings, e.g., long internal waves in a density-stratified ocean, acoustic waves on a crystal lattice, ion-acoustic

This work was supported by National Nature Science Foundation of China under grant numbers 10801055 and 10901057, and by Doctoral Program of Ministry of Education of China under grant number 200805611026. 
waves in a plasma. The mathematical theory behind the KdV equation is a rich, active and interesting research topic. It is the first discovered integrable nonlinear partial differential equation, and there have been lots of works on its exact solutions, wellposedness and long time behavior, etc.

The wellposedness for periodic initial value problem and Cauchy problem of the $\mathrm{KdV}$ equation in Sobolev spaces has been investigated by many authors. Bourgain [2] proved that the periodic initial value problem is locally uniformly wellposed in $L^{2}(\mathbf{T})$ by the Fourier restriction norm method, and thus is globally uniformly wellposed due to the $L^{2}(\mathbf{T})$ conservation law. Later, Kenig et al. [3,4] extended the local result to the range $s>-1 / 2$, and at the same time they proved the Cauchy problem is locally uniformly wellposed in $H^{s}(\mathbf{R})$ for $s>-3 / 4$. Christ et al. [5] extended the local result to the endpoint $s=-3 / 4$, using a modified Miura transform and the modified $\mathrm{KdV}$ theory. Moreover, the KdV equation is not uniformly wellposed in $H^{s}(\mathbf{T})$ for $s<-1 / 2$ and in $H^{s}(\mathbf{R})$ for $s<-3 / 4$. We refer the readers to [6] and [5] for the complex-valued and real-valued case, respectively. Colliander et al. $[7,8]$ proved that the $\mathrm{KdV}$ equation in the real setting is globally uniformly wellposed in $H^{s}(\mathbf{T})$ for $s \geq-1 / 2$ and in $H^{s}(\mathbf{R})$ for $s>-3 / 4$ by using the Imethod. It is worth noting that the global solutions obtained by the I-method increase polynomially in time. Guo [9] proved a stronger local uniform wellposedness result in $H^{-3 / 4}(\mathbf{R})$ (compared with [5]) and extended it to a global uniform one by the I-method. Using the complete integrability of the equation, Kappeler and Topalov [10] proved the $\mathrm{KdV}$ is globally $C^{0}$-wellposed in $H^{s}(\mathbf{T})$ for $s \geq-1$. Incidentally, it is an interesting phenomenon, that an equation can be globally wellposed, but not in a uniform way [11].

In many real situations, one cannot neglect energy dissipation mechanisms and external excitation, especially for the long time behavior. Therefore, many works considered the global attractor for the damped forced $\mathrm{KdV}$ equation, e.g., [12-17]. Here we only mention the most recent results. Goubet [15] proved the existence of the global attractor for Eq. (1.1) in $\dot{L}^{2}(\mathbf{T})$ and the compactness in $H^{3}(\mathbf{T})$, while Goubet and Rosa [16] obtained the similar result for the non-periodic case. The global solution to Eq. (1.1) obtained by the Imethod increases polynomially while the damping term $\gamma u$ may prevent the solution from increasing unlimitedly, therefore we can expect the existence of the global attractor in Sobolev spaces of negative index. Tsugawa [17] proved that the global attractor in $\dot{H}^{s}(\mathbf{T})$ for $s>-3 / 8$ exists and is compact in $H^{3}(\mathbf{T})$ under the assumption that the forcing term $f \in \dot{L}^{2}(\mathbf{T})$. In this paper, we follow the framework of [8] and [17] and aim to lower the index $s$ down to the critical case $s=-1 / 2$. Comparing with [17], we make a higher degree correction to the modified energy as in [8]. The main result of this paper is as follows.

Theorem 1.1. Let $-1 / 2 \leq s<0, \gamma>0$ and $f \in \dot{L}^{2}(\mathbf{T})$. Then the solution operator $\{S(t)\}_{t \in \mathbf{R}}$ associated to Eq. (1.1) possesses a global attractor $\mathscr{A}$ in $\dot{H}^{s}(\mathbf{T})$, which is compact in $H^{3}(\mathbf{T})$. 
To prove the existence of the global attractor in $\dot{H}^{s}(\mathbf{T})$ for $s<0$, by Theorem 1.1 of Chapter I in [18], we need only to split the solution into two parts, one of which is in $\dot{L}^{2}(\mathbf{T})$ and thus is compact in $\dot{H}^{s}(\mathbf{T})$, and the other decays to zero in $\dot{H}^{s}(\mathbf{T})$. It is easy to show that the global attractor is the same as the one in $\dot{L}^{2}(\mathbf{T})$. The proof of Theorem 1.1 is mainly relied on the following theorem.

Theorem 1.2. Assume $-1 / 2 \leq s<0, \gamma>0$ and $f \in \dot{L}^{2}(\mathbf{T})$. Then there exist a semigroup $S(t)$ and maps $M_{1}(t)$ and $M_{2}(t)$ such that for initial data $u_{0} \in \dot{H}^{s}(\mathbf{T}), S(t) u_{0}$ is the unique solution to the periodic initial value problem of Eq. (1.1), and satisfies

$$
\begin{aligned}
& S(t) u_{0}=M_{1}(t) u_{0}+M_{2}(t) u_{0}, \\
& \sup _{t>T_{1}}\left\|M_{1}(t) u_{0}\right\|_{L^{2}} \leq K
\end{aligned}
$$

and for $t>T_{1}$,

$$
\left\|M_{2}(t) u_{0}\right\|_{H^{s}} \leq K \exp \left(-\gamma\left(t-T_{1}\right)\right)
$$

where the constant $K$ depends only on $\|f\|_{L^{2}}$ and $\gamma$, and $T_{1}$ depends on $\|f\|_{L^{2}}, \gamma$ and $\left\|u_{0}\right\|_{H^{s}}$.

This paper is arranged as follows. In Sect. 2, we rescale Eq. (1.1) and present the local wellposedness for the rescaled equation. In Sect. 3, we prove an a priori estimate. In Sect. 4, we present the proof of Theorem 1.2.

\section{Local wellposedness result}

In this section, we first rescale Eq. (1.1) and then present the local wellposedness for the rescaled equation.

By setting

$$
\begin{aligned}
v(x, t) & =\lambda^{-2} u\left(\lambda^{-1} x, \lambda^{-3} t\right), \\
g(x) & =\lambda^{-2} f\left(\lambda^{-1} x\right), \quad x \in[0, \lambda], \quad t \in \mathbf{R},
\end{aligned}
$$

we rewrite Eq. (1.1) as

$$
\partial_{t} v+\partial_{x}^{3} v+\frac{1}{2} \partial_{x} v^{2}+\lambda^{-3} \gamma v=\lambda^{-3} g, \quad x \in[0, \lambda], \quad t>0 .
$$

We supply Eq. (2.1) with the initial data

$$
v\left(x, t_{0}\right)=v_{t_{0}}(x)=\lambda^{-2} u\left(\lambda^{-1} x, \lambda^{-3} t_{0}\right) .
$$

Next we introduce some notations. The notations $a+$ and $a$ - denote, respectively, $a+\varepsilon$ and $a-\varepsilon$ for an arbitrary small positive number $\varepsilon$. Define $(d k)_{\lambda}$ to be normalized counting measure on $A \subseteq \mathbf{Z} / \lambda=\{n / \lambda: n \in \mathbf{Z}\}$ as follows:

$$
\int_{A} a(k)(d k)_{\lambda}=\frac{1}{\lambda} \sum_{k \in A} a(k) .
$$


Define the Fourier transform in $x$ of a function $g$ defined on $[0, \lambda]$ by

$$
\widehat{g}(k)=\int_{0}^{\lambda} g(x) e^{-2 \pi i k x} d x, \quad k \in \mathbf{Z} / \lambda .
$$

The usual Plancherel identity holds

$$
\|g\|_{L^{2}([0, \lambda])}=\|\widehat{g}\|_{L^{2}\left((d k)_{\lambda}\right)}
$$

Define the Sobolev space $H^{s}([0, \lambda])$ with the norm

$$
\|g\|_{H^{s}([0, \lambda])}=\left\|\langle k\rangle^{s} \widehat{g}\right\|_{L^{2}\left((d k)_{\lambda}\right)},
$$

where $\langle\cdot\rangle=1+|\cdot|$.

For $N>1$, we introduce Fourier restriction operators $P_{N}$ and $P^{N}$, defined as

$$
\begin{aligned}
P_{N} g(x) & =\int_{|k| \leq N} \widehat{g}(k) e^{2 \pi i k x}(d k)_{\lambda}, \\
P^{N} g(x) & =\int_{|k|>N} \widehat{g}(k) e^{2 \pi i k x}(d k)_{\lambda}, \quad x \in[0, \lambda] .
\end{aligned}
$$

For a function $v=v(x, t)$ on $[0, \lambda] \times \mathbf{R}$, define its space-time Fourier transform in $x$ and $t$ by

$$
\widehat{v}(k, \tau)=\int_{\mathbf{R}} \int_{0}^{\lambda} v(x, t) e^{-2 \pi i k x-2 \pi i \tau t} d x d t, \quad k \in \mathbf{Z} / \lambda, \tau \in \mathbf{R} .
$$

For $s, b \in \mathbf{R}$, we define $X_{s, b}$ to be the function space with respect to the norm

$$
\|v\|_{X_{s, b}}=\left\|\langle k\rangle^{s}\left\langle\tau-k^{3}\right\rangle^{b} \widehat{v}(k, \tau)\right\|_{L^{2}\left((d k)_{\lambda} d \tau\right)} .
$$

To control the $L_{t}^{\infty} H_{x}^{s}$ norm and ensure continuity of the time flow of the solution, we introduce the slightly smaller space $Y_{s}$ defined via the norm

$$
\|v\|_{Y_{s}}=\|v\|_{X_{s, \frac{1}{2}}}+\left\|\langle k\rangle^{s} \widehat{v}(k, \tau)\right\|_{L^{2}\left((d k)_{\lambda}\right) L^{1}(d \tau)} .
$$

For a given time interval $I$, define the function spaces $X_{s, b}^{I}$ and $Y_{s}^{I}$ to be the restriction of $X_{s, b}$ and $Y_{s}$ on $[0, \lambda] \times I$ with norms

$$
\|v\|_{X_{s, b}^{I}}=\inf \left\{\|V\|_{X_{s, b}}: V \in X_{s, b},\left.V\right|_{[0, \lambda] \times I}=v\right\}
$$

and

$$
\|v\|_{Y_{s}^{I}}=\inf \left\{\|V\|_{Y_{s}}: V \in Y_{s},\left.V\right|_{[0, \lambda] \times I}=v\right\}
$$

respectively.

Let $s<0$ and $N>1$ be fixed. Define Fourier multiplier operator $I$ acting on $f \in H^{s}(\mathbf{T})$ by

$$
\widehat{I f}(n)=m(n) \widehat{f}(n), \quad n \in \mathbf{Z},
$$

where $m$ is a smooth radially monotone even function and satisfies

$$
m(\xi)= \begin{cases}1, & |\xi| \leq N \\ \left(\frac{|\xi|}{N}\right)^{s}, & |\xi| \geq 2 N\end{cases}
$$


It is easy to show that the following inequalities hold:

$$
\begin{aligned}
\|f\|_{H^{s}} & \leq\|I f\|_{L^{2}} \leq C N^{-s}\|f\|_{H^{s}} \\
\|u\|_{Y_{s}} & \leq\|I u\|_{Y_{0}} \leq C N^{-s}\|u\|_{Y_{s}} .
\end{aligned}
$$

Set $f_{\lambda}(x)=\lambda^{-2} f\left(\lambda^{-1} x\right), x \in[0, \lambda]$, and we have

$$
\lambda \widehat{f_{\lambda}}(n / \lambda)=\widehat{f}(n), \quad n \in \mathbf{Z}, \quad \lambda^{\frac{3}{2}}\left\|f_{\lambda}\right\|_{L^{2}([0, \lambda])}=\|f\|_{L^{2}(\mathbf{T})} .
$$

We define the rescaled operator $I^{\prime}$ as follows:

$$
\widehat{I^{\prime} g}(k)=m^{\prime}(k) \widehat{g}(k), \quad k \in \mathbf{Z} / \lambda,
$$

where

$$
m^{\prime}(\eta)=m(\lambda \eta)=\left\{\begin{array}{ll}
1, & |\eta| \leq N^{\prime} ; \\
\left(\frac{|\eta|}{N^{\prime}}\right)^{s}, & |\eta| \geq 2 N^{\prime},
\end{array} \quad N^{\prime}=N / \lambda\right.
$$

We have

$$
\lambda^{\frac{3}{2}}\left\|I^{\prime} f_{\lambda}\right\|_{L^{2}([0, \lambda])}=\|I f\|_{L^{2}(\mathbf{T})} .
$$

Next, we present the local wellposedness result for Eq. (2.1) in the following, and for the proof refer the readers to [8] or [17].

Theorem 2.1. Assume $s \geq-1 / 2, g \in \dot{H}^{s}([0, \lambda])$, then the $\lambda$-periodic initial value problem of Eq. (2.1) is locally wellposed in $\dot{H}^{s}([0, \lambda])$. More precisely, Eq. (2.1) with initial data $v_{t_{0}} \in \dot{H}^{s}([0, \lambda])$ possesses a unique solution $v \in$ $C\left(\left[t_{0}, t_{0}+\delta\right] ; \dot{H}^{s}([0, \lambda])\right)$ with the lifespan

$$
\delta \sim\left(\left\|I^{\prime} v_{t_{0}}\right\|_{L^{2}}+\lambda^{-3}\left\|I^{\prime} g\right\|_{L^{2}}+\gamma \lambda^{-3}\right)^{-\alpha}
$$

for some $\alpha>0$, and the local solution $v$ depends continuously on the initial data $v_{t_{0}}$, i.e., the mapping $v_{t_{0}} \mapsto v$ belongs to $C\left(\dot{H}^{s}([0, \lambda]) ; C\left(\left[t_{0}, t_{0}+\delta\right] ; \dot{H}^{s}([0, \lambda])\right)\right)$. Moreover, the solution satisfies

$$
\begin{aligned}
& \left\|I^{\prime} v\right\|_{Y_{0}} \leq C\left(\left\|I^{\prime} v_{t_{0}}\right\|_{L^{2}}+\lambda^{-3}\left\|I^{\prime} g\right\|_{L^{2}}\right) ; \\
& \sup _{t \in\left[t_{0}, t_{0}+\delta\right]}\left\|I^{\prime} v(t)\right\|_{L^{2}} \leq C\left(\left\|I^{\prime} v_{t_{0}}\right\|_{L^{2}}+\lambda^{-3}\left\|I^{\prime} g\right\|_{L^{2}}\right) .
\end{aligned}
$$

\section{A Priori estimate}

In this section, we prove an a priori estimate. First we introduce some notations.

Definition 3.1. A $j$-multiplier is a function $M:(\mathbf{Z} / \lambda)^{j} \mapsto$ C. A $j$-multiplier $M$ is symmetric if $M\left(\sigma\left(k_{1}, \ldots, k_{j}\right)\right)=M\left(k_{1}, \ldots, k_{j}\right)$ for all $\sigma \in S_{j}$, the group of all permutations on $j$ objects. The symmetrization of a $j$-multiplier $M$ is

$$
[M]_{s y m}\left(k_{1}, \ldots, k_{j}\right)=\frac{1}{j !} \sum_{\sigma \in S_{j}} M\left(\sigma\left(k_{1}, \ldots, k_{j}\right)\right) .
$$


Definition 3.2. A $j$-multiplier $M$ generates $j$-linear functional on $j$ functions $v_{1}, \ldots, v_{j}$ defined on $[0, \lambda]$,

$$
\begin{aligned}
& \Lambda_{j}\left(M ; v_{1}, \ldots, v_{j}\right) \\
& \quad=\int_{k_{1}+\cdots+k_{j}=0} M\left(k_{1}, \ldots, k_{j}\right) \widehat{v_{1}}\left(k_{1}\right) \ldots \widehat{v_{j}}\left(k_{j}\right) \quad\left(d k_{1}\right)_{\lambda} \ldots\left(d k_{j-1}\right)_{\lambda} .
\end{aligned}
$$

For simplicity, note $\Lambda_{j}(M)=\Lambda_{j}(M ; v, \ldots, v)$. If $M$ is a symmetric $j$-multiplier, $\Lambda_{j}(M)$ is a symmetric $j$-functional.

To estimate the increase of the modified energy, we calculate the partial derivative in time of the symmetric $j$-functional $\Lambda_{j}(M)$ assuming the function $v$ satisfies Eq. (2.1).

Proposition 3.3. Suppose that $M$ is a symmetric $j$-multiplier and $v$ satisfies Eq. (2.1). Then

$$
\begin{aligned}
\frac{d}{d t} \Lambda_{j}(M)= & -j \gamma \lambda^{-3} \Lambda_{j}(M)+\Lambda_{j}\left(\alpha_{j} M\right)+j \lambda^{-3} \Lambda_{j}(M ; v, \ldots, v, g) \\
& -i \frac{j}{2} \Lambda_{j+1}\left(M\left(k_{1}, \ldots, k_{j-1}, k_{j}+k_{j+1}\right)\left(k_{j}+k_{j+1}\right)\right),
\end{aligned}
$$

where

$$
\alpha_{j}=i\left(k_{1}^{3}+\cdots+k_{j}^{3}\right) .
$$

We define the modified energy $E_{I^{\prime}}^{2}(t)$ by

$$
E_{I^{\prime}}^{2}(t)=\left\|I^{\prime} v(t)\right\|_{L^{2}}^{2} .
$$

By Plancherel identity and the fact that $m^{\prime}$ and $v$ are real-valued, we have

$$
E_{I^{\prime}}^{2}(t)=\Lambda_{2}\left(\sigma_{2}\right)
$$

where $\sigma_{2}\left(k_{1}, k_{2}\right)=m^{\prime}\left(k_{1}\right) m^{\prime}\left(k_{2}\right), k_{1}, k_{2} \in \mathbf{Z} / \lambda$. By using (3.1), we have

$$
\begin{aligned}
\frac{d}{d t} E_{I^{\prime}}^{2}(t)= & -2 \gamma \lambda^{-3} \Lambda_{2}\left(\sigma_{2}\right)+\Lambda_{2}\left(\alpha_{2} \sigma_{2}\right)+2 \lambda^{-3} \Lambda_{2}\left(\sigma_{2} ; v, g\right) \\
& -i \Lambda_{3}\left(\sigma_{2}\left(k_{1}, k_{2}+k_{3}\right)\left(k_{2}+k_{3}\right)\right) .
\end{aligned}
$$

Noticing the second term vanishes and symmetrizing the last term, we get

$$
\begin{aligned}
\frac{d}{d t} E_{I^{\prime}}^{2}(t)= & -2 \gamma \lambda^{-3} E_{I^{\prime}}^{2}(t)+2 \lambda^{-3} \Lambda_{2}\left(\sigma_{2} ; v, g\right) \\
& +\Lambda_{3}\left(-i\left[\sigma_{2}\left(k_{1}, k_{2}+k_{3}\right)\left(k_{2}+k_{3}\right)\right]_{s y m}\right) .
\end{aligned}
$$

Note

$$
M_{3}\left(k_{1}, k_{2}, k_{3}\right)=-i\left[\sigma_{2}\left(k_{1}, k_{2}+k_{3}\right)\left(k_{2}+k_{3}\right)\right]_{s y m} .
$$

Form the new modified energy

$$
E_{I^{\prime}}^{3}(t)=E_{I^{\prime}}^{2}(t)+\Lambda_{3}\left(\sigma_{3}\right),
$$


where the symmetric 3-multiplier $\sigma_{3}$ will be chosen momentarily to achieve a cancellation. By using (3.1) again, we have

$$
\begin{aligned}
\frac{d}{d t} E_{I^{\prime}}^{3}(t)= & -2 \gamma \lambda^{-3} E_{I^{\prime}}^{3}(t)+\Lambda_{3}\left(M_{3}\right)+2 \lambda^{-3} \Lambda_{2}\left(\sigma_{2} ; v, g\right) \\
& -\gamma \lambda^{-3} \Lambda_{3}\left(\sigma_{3}\right)+\Lambda_{3}\left(\alpha_{3} \sigma_{3}\right)+3 \lambda^{-3} \Lambda_{3}\left(\sigma_{3} ; v, v, g\right) \\
& +\Lambda_{4}\left(-\frac{3}{2} i\left[\sigma_{3}\left(k_{1}, k_{2}, k_{3}+k_{4}\right)\left(k_{3}+k_{4}\right)\right]_{s y m}\right)
\end{aligned}
$$

By choosing

$$
\sigma_{3}=-\frac{M_{3}}{\alpha_{3}}
$$

to force the term $\Lambda_{3}\left(M_{3}\right)$ and $\Lambda_{3}\left(\alpha_{3} \sigma_{3}\right)$ in (3.3) to cancel, we have

$$
\begin{aligned}
\frac{d}{d t} E_{I^{\prime}}^{3}(t)= & -2 \gamma \lambda^{-3} E_{I^{\prime}}^{3}(t)+2 \lambda^{-3} \Lambda_{2}\left(\sigma_{2} ; v, g\right)+3 \lambda^{-3} \Lambda_{3}\left(\sigma_{3} ; v, v, g\right) \\
& -\gamma \lambda^{-3} \Lambda_{3}\left(\sigma_{3}\right)+\Lambda_{4}\left(M_{4}\right)
\end{aligned}
$$

where

$$
M_{4}\left(k_{1}, k_{2}, k_{3}, k_{4}\right)=-\frac{3}{2} i\left[\sigma_{3}\left(k_{1}, k_{2}, k_{3}+k_{4}\right)\left(k_{3}+k_{4}\right)\right]_{s y m} .
$$

Upon defining

$$
E_{I^{\prime}}^{4}(t)=E_{I^{\prime}}^{3}(t)+\Lambda_{4}\left(\sigma_{4}\right)
$$

with

$$
\sigma_{4}=-\frac{M_{4}}{\alpha_{4}}
$$

we obtain

$$
\begin{aligned}
\frac{d}{d t} E_{I^{\prime}}^{4}(t)= & -2 \gamma \lambda^{-3} E_{I^{\prime}}^{4}(t)+2 \lambda^{-3} \Lambda_{2}\left(\sigma_{2} ; v, g\right)+3 \lambda^{-3} \Lambda_{3}\left(\sigma_{3} ; v, v, g\right) \\
& +4 \lambda^{-3} \Lambda_{4}\left(\sigma_{4} ; v, v, v, g\right)-\gamma \lambda^{-3} \Lambda_{3}\left(\sigma_{3}\right) \\
& -2 \gamma \lambda^{-3} \Lambda_{4}\left(\sigma_{4}\right)+\Lambda_{5}\left(M_{5}\right)
\end{aligned}
$$

where

$$
M_{5}\left(k_{1}, \ldots, k_{5}\right)=-2 i\left[\sigma_{4}\left(k_{1}, k_{2}, k_{3}, k_{4}+k_{5}\right)\left(k_{4}+k_{5}\right)\right]_{s y m} .
$$

From Lemmas 6.1 and 8.2 in [8], we borrow the following results.

Lemma 3.4. It holds that

$$
\begin{aligned}
& \left|\Lambda_{3}\left(\sigma_{3} ; v_{1}, v_{2}, v_{3}\right)\right| \leq C \prod_{j=1}^{3}\left\|I^{\prime} v_{j}(t)\right\|_{L^{2}} ; \\
& \left|\Lambda_{4}\left(\sigma_{4} ; v_{1}, v_{2}, v_{3}, v_{4}\right)\right| \leq C \prod_{j=1}^{4}\left\|I^{\prime} v_{j}(t)\right\|_{L^{2}} .
\end{aligned}
$$


Lemma 3.5. If $m^{\prime}$ is of the form (2.6) with $-1 / 2 \leq s<0$, it holds that

$$
\left|\int_{0}^{\delta} \Lambda_{5}\left(M_{5}\right) d t\right| \leq C \lambda^{0+} N^{-\frac{5}{2}+}\left\|I^{\prime} v\right\|_{Y_{0}}^{5} .
$$

Lemma 3.6. Let $v$ be a solution to Eq. (2.1) with initial data $v_{0} \in \dot{H}^{s}([0, \lambda])$ on $\left[0, T^{\prime}\right]$, and

$$
\sup _{0 \leq t \leq T^{\prime}}\left\|I^{\prime} v(t)\right\|_{L^{2}} \ll 1
$$

Then we have

$$
\begin{aligned}
\left\|I^{\prime} v\left(T^{\prime}\right)\right\|_{L^{2}}^{2} \exp \left(2 \gamma \lambda^{-3} T^{\prime}\right) \leq & C_{1}\left(\left\|I^{\prime} v_{0}\right\|_{L^{2}}^{2}+\gamma^{-2}\left\|I^{\prime} g\right\|_{L^{2}}^{2} \exp \left(2 \gamma \lambda^{-3} T^{\prime}\right)\right. \\
& \left.+\left|\int_{0}^{T^{\prime}} \Lambda_{5}\left(M_{5}\right) \exp \left(2 \gamma \lambda^{-3} t\right) d t\right|\right)
\end{aligned}
$$

Proof. The proof is similar to Lemma 3.6 in [17]. From (3.5), we have

$$
\begin{aligned}
\frac{d}{d t}( & \left.E_{I^{\prime}}^{4}(t) \exp \left(2 \gamma \lambda^{-3} t\right)\right) \\
= & 2 \lambda^{-3} \Lambda_{2}\left(\sigma_{2} ; v, g\right) \exp \left(2 \gamma \lambda^{-3} t\right)+3 \lambda^{-3} \Lambda_{3}\left(\sigma_{3} ; v, v, g\right) \exp \left(2 \gamma \lambda^{-3} t\right) \\
& +4 \lambda^{-3} \Lambda_{4}\left(\sigma_{4} ; v, v, v, g\right) \exp \left(2 \gamma \lambda^{-3} t\right)-\gamma \lambda^{-3} \Lambda_{3}\left(\sigma_{3}\right) \exp \left(2 \gamma \lambda^{-3} t\right) \\
& -2 \gamma \lambda^{-3} \Lambda_{4}\left(\sigma_{4}\right) \exp \left(2 \gamma \lambda^{-3} t\right)+\Lambda_{5}\left(M_{5}\right) \exp \left(2 \gamma \lambda^{-3} t\right) .
\end{aligned}
$$

Integrating (3.8) on $\left[0, T^{\prime}\right]$, by Lemma 3.4 , we have

$$
\begin{aligned}
& \left|E_{I^{\prime}}^{4}\left(T^{\prime}\right) \exp \left(2 \gamma \lambda^{-3} T^{\prime}\right)-E_{I^{\prime}}^{4}(0)\right| \\
& \leq \frac{1}{2 \gamma} C\left\|I^{\prime} g\right\|_{L^{2}} \exp \left(2 \gamma \lambda^{-3} T^{\prime}\right) \\
& \quad \times \sup _{0 \leq t \leq T^{\prime}}\left(2\left\|I^{\prime} v(t)\right\|_{L^{2}}+3\left\|I^{\prime} v(t)\right\|_{L^{2}}^{2}+4\left\|I^{\prime} v(t)\right\|_{L^{2}}^{3}\right) \\
& \quad+\frac{1}{2} C \exp \left(2 \gamma \lambda^{-3} T^{\prime}\right) \sup _{0 \leq t \leq T^{\prime}}\left(\left\|I^{\prime} v(t)\right\|_{L^{2}}^{3}+2\left\|I^{\prime} v(t)\right\|_{L^{2}}^{4}\right) \\
& \quad+\left|\int_{0}^{T^{\prime}} \Lambda_{5}\left(M_{5}\right) \exp \left(2 \gamma \lambda^{-3} t\right) d t\right| .
\end{aligned}
$$

Noticing (3.6), we have

$$
\begin{aligned}
E_{I^{\prime}}^{4}\left(T^{\prime}\right) & \geq\left\|I^{\prime} v\left(T^{\prime}\right)\right\|_{L^{2}}^{2}-C\left(\left\|I^{\prime} v\left(T^{\prime}\right)\right\|_{L^{2}}^{3}+\left\|I^{\prime} v\left(T^{\prime}\right)\right\|_{L^{2}}^{4}\right) \\
& \geq \frac{1}{2}\left\|I^{\prime} v\left(T^{\prime}\right)\right\|_{L^{2}}^{2}
\end{aligned}
$$

and

$$
E_{I^{\prime}}^{4}(0) \leq\left\|I^{\prime} v_{0}\right\|_{L^{2}}^{2}+C\left(\left\|I^{\prime} v_{0}\right\|_{L^{2}}^{3}+\left\|I^{\prime} v_{0}\right\|_{L^{2}}^{4}\right) \leq 2\left\|I^{\prime} v_{0}\right\|_{L^{2}}^{2} .
$$


By Cauchy-Schwarz inequality, we have

$$
\begin{aligned}
\frac{1}{2 \gamma} C & \left\|I^{\prime} g\right\|_{L^{2}} \exp \left(2 \gamma \lambda^{-3} T^{\prime}\right) \\
& \times \sup _{0 \leq t \leq T^{\prime}}\left(2\left\|I^{\prime} v(t)\right\|_{L^{2}}+3\left\|I^{\prime} v(t)\right\|_{L^{2}}^{2}+4\left\|I^{\prime} v(t)\right\|_{L^{2}}^{3}\right) \\
& +\frac{1}{2} C \exp \left(2 \gamma \lambda^{-3} T^{\prime}\right) \sup _{0 \leq t \leq T^{\prime}}\left(\left\|I^{\prime} v(t)\right\|_{L^{2}}^{3}+2\left\|I^{\prime} v(t)\right\|_{L^{2}}^{4}\right) \\
\leq & \frac{1}{\gamma^{2}} C\left\|I^{\prime} g\right\|_{L^{2}}^{2} \exp \left(2 \gamma \lambda^{-3} T^{\prime}\right)+\frac{5}{C} \exp \left(2 \gamma \lambda^{-3} T^{\prime}\right) \underset{0 \leq t \leq T^{\prime}}{\sup }\left\|I^{\prime} v(t)\right\|_{L^{2}}^{2} \\
\leq & \frac{1}{\gamma^{2}} C\left\|I^{\prime} g\right\|_{L^{2}}^{2} \exp \left(2 \gamma \lambda^{-3} T^{\prime}\right)+\frac{1}{4} \exp \left(2 \gamma \lambda^{-3} T^{\prime}\right)\left\|I^{\prime} v\left(T^{\prime}\right)\right\|_{L^{2}}^{2},
\end{aligned}
$$

for some large $C$. Thus we obtain (3.7).

Next we establish an a priori estimate for Eq. (2.1).

Proposition 3.7. Let $-1 / 2 \leq s<0, T^{\prime}>0$ be given and $v$ be a solution to Eq. (2.1) with initial data $v_{0} \in \dot{H}^{s}([0, \lambda])$ on $\left[0, T^{\prime}\right]$. Assume $\lambda^{3} \geq$ $\gamma, \lambda^{0-} N^{\prime \frac{5}{2}-} \geq C_{2} T^{\prime}$ and

$$
\left\|I^{\prime} v_{0}\right\|_{L^{2}}^{2}+\gamma^{-2}\left\|I^{\prime} g\right\|_{L^{2}}^{2} \exp \left(2 \gamma \lambda^{-3} T^{\prime}\right) \leq C_{3} \ll 1 \text {. }
$$

Then we have

$$
\begin{aligned}
& \left\|I^{\prime} v\left(T^{\prime}\right)\right\|_{L^{2}}^{2} \exp \left(2 \gamma \lambda^{-3} T^{\prime}\right) \\
& \quad \leq C_{4}\left(\left\|I^{\prime} v_{0}\right\|_{L^{2}}^{2}+\gamma^{-2}\left\|I^{\prime} g\right\|_{L^{2}}^{2} \exp \left(2 \gamma \lambda^{-3} T^{\prime}\right)\right) .
\end{aligned}
$$

Proof. Let $\delta$ be the lifespan of the local solution obtained in Theorem 2.1. Choose $l \in \mathbf{N}$ such that $l \delta=T^{\prime}$. For $0 \leq k \leq l$, we prove

$$
\begin{aligned}
& \left\|I^{\prime} v(k \delta)\right\|_{L^{2}}^{2} \exp \left(2 \gamma \lambda^{-3} k \delta\right) \\
& \quad \leq 2 C_{1}\left(\left\|I^{\prime} v_{0}\right\|_{L^{2}}^{2}+\gamma^{-2}\left\|I^{\prime} g\right\|_{L^{2}}^{2} \exp \left(2 \gamma \lambda^{-3} k \delta\right)\right)
\end{aligned}
$$

by induction. For $k=0,(3.11)$ holds obviously. We assume (3.11) holds for $0 \leq k \leq j$, where $0 \leq j \leq l-1$. Next we aim to prove that (3.11) holds for $k=j+1$.

From (2.10), we have

$$
\left\|I^{\prime} v((j+1) \delta)\right\|_{L^{2}} \leq C\left(\left\|I^{\prime} v(j \delta)\right\|_{L^{2}}+\lambda^{-3}\left\|I^{\prime} g\right\|_{L^{2}}\right) .
$$

From (3.11) for $k=j$ and (3.9), we have

$$
\left\|I^{\prime} v(j \delta)\right\|_{L^{2}}^{2} \leq 2 C_{1} C_{3} .
$$

For $\lambda^{3} \geq \gamma$, we have

$$
\left(\lambda^{-3}\left\|I^{\prime} g\right\|_{L^{2}}\right)^{2} \leq \gamma^{-2}\left\|I^{\prime} g\right\|_{L^{2}}^{2} \leq C_{3} .
$$

Noticing $C_{3} \ll 1$, we have

$$
\left\|I^{\prime} v((j+1) \delta)\right\|_{L^{2}}^{2} \ll 1 .
$$


From Lemma 3.6, we have

$$
\begin{aligned}
& \left\|I^{\prime} v((j+1) \delta)\right\|_{L^{2}}^{2} \exp \left(2 \gamma \lambda^{-3}(j+1) \delta\right) \\
& \leq C_{1}\left(\left\|I^{\prime} v_{0}\right\|_{L^{2}}^{2}+\gamma^{-2}\left\|I^{\prime} g\right\|_{L^{2}}^{2} \exp \left(2 \gamma \lambda^{-3}(j+1) \delta\right)\right. \\
& \left.\quad+\left|\int_{0}^{(j+1) \delta} \Lambda_{5}\left(M_{5}\right) \exp \left(2 \gamma \lambda^{-3} t\right) d t\right|\right) .
\end{aligned}
$$

Therefore, it suffices to prove

$$
\begin{aligned}
& \left|\int_{0}^{(j+1) \delta} \Lambda_{5}\left(M_{5}\right) \exp \left(2 \gamma \lambda^{-3} t\right) d t\right| \\
& \quad \leq\left\|I^{\prime} v_{0}\right\|_{L^{2}}^{2}+\gamma^{-2}\left\|I^{\prime} g\right\|_{L^{2}}^{2} \exp \left(2 \gamma \lambda^{-3}(j+1) \delta\right) .
\end{aligned}
$$

In fact, by Lemma 3.5, we have

$$
\begin{aligned}
& \left|\int_{0}^{(j+1) \delta} \Lambda_{5}\left(M_{5}\right) \exp \left(2 \gamma \lambda^{-3} t\right) d t\right| \\
& \quad \leq \sum_{k=0}^{j} \exp \left(2 \gamma \lambda^{-3}(k+1) \delta\right)\left|\int_{k \delta}^{(k+1) \delta} \Lambda_{5}\left(M_{5}\right) d t\right| \\
& \quad \leq C \lambda^{0+} N^{\prime-\frac{5}{2}+} \sum_{k=0}^{j}\left\|I^{\prime} v\right\|_{Y_{0}^{I_{k}}}^{5} \exp \left(2 \gamma \lambda^{-3}(k+1) \delta\right) \\
& \quad \leq C\left(C_{2} T^{\prime}\right)^{-1} \sum_{k=0}^{j}\left\|I^{\prime} v\right\|_{Y_{0}^{I_{k}}}^{5} \exp \left(2 \gamma \lambda^{-3}(k+1) \delta\right),
\end{aligned}
$$

where $I_{k}=[k \delta,(k+1) \delta], k=0,1, \ldots, j$. From $(2.9)$, we have

$$
\left\|I^{\prime} v\right\|_{Y_{0}^{I_{k}}}^{5} \leq C\left(\left\|I^{\prime} v(k \delta)\right\|_{L^{2}}^{5}+\left(\lambda^{-3}\left\|I^{\prime} g\right\|_{L^{2}}\right)^{5}\right) .
$$

From (3.11) for $0 \leq k \leq j$, we have

$$
\begin{aligned}
& \left\|I^{\prime} v(k \delta)\right\|_{L^{2}}^{5} \exp \left(2 \gamma \lambda^{-3}(k+1) \delta\right) \\
& \quad \leq\left(\left\|I^{\prime} v(k \delta)\right\|_{L^{2}}^{2} \exp \left(2 \gamma \lambda^{-3} k \delta\right)\right)^{\frac{5}{2}} \\
& \quad \leq C C_{1}^{\frac{5}{2}} C_{3}^{\frac{3}{2}}\left(\left\|I^{\prime} v_{0}\right\|_{L^{2}}^{2}+\gamma^{-2}\left\|I^{\prime} g\right\|_{L^{2}}^{2} \exp \left(2 \gamma \lambda^{-3} k \delta\right)\right)
\end{aligned}
$$

and

$$
\begin{aligned}
& \left(\lambda^{-3}\left\|I^{\prime} g\right\|_{L^{2}}\right)^{5} \exp \left(2 \gamma \lambda^{-3}(k+1) \delta\right) \\
& \quad \leq C C_{1}^{\frac{5}{2}} C_{3}^{\frac{3}{2}}\left(\left\|I^{\prime} v_{0}\right\|_{L^{2}}^{2}+\gamma^{-2}\left\|I^{\prime} g\right\|_{L^{2}}^{2} \exp \left(2 \gamma \lambda^{-3} k \delta\right)\right) .
\end{aligned}
$$

By taking $C_{2}$ large enough, we have

$$
2 C(l+1)\left(C_{2} T^{\prime}\right)^{-1} C_{1}^{\frac{5}{2}} C_{3}^{\frac{3}{2}} \leq 2 C\left(C_{2} \delta\right)^{-1} C_{1}^{\frac{5}{2}} C_{3}^{\frac{3}{2}}<1,
$$

and thus obtain (3.13). 
Noticing $\lambda^{3}\left\|I^{\prime} g\right\|_{L^{2}([0, \lambda])}^{2}=\|I f\|_{L^{2}(\mathbf{T})}^{2}$ and taking $\lambda=N^{(5-2 \varepsilon) / 11}, N^{\prime}=$ $N / \lambda$ and $T^{\prime}=\lambda^{3} T$ in Proposition 3.7 for $0<\varepsilon<(22 s+15) / 6$, we obtain an a priori estimate for Eq. (1.1).

Proposition 3.8. Let $-1 / 2 \leq s<0, T>0$ be given and $u$ be a solution to Eq. (1.1) with initial data $u_{0} \in \dot{H}^{s}(\mathbf{T})$ on $[0, T]$. Assume $N^{3(5-2 \varepsilon) / 11} \geq \gamma$, $N^{\varepsilon-} \geq C_{2} T$ and

$$
\left\|I u_{0}\right\|_{L^{2}}^{2}+\gamma^{-2}\|I f\|_{L^{2}}^{2} \exp (2 \gamma T) \leq C_{3} N^{\frac{3(5-2 \varepsilon)}{11}}, \quad C_{3} \ll 1
$$

for $0<\varepsilon<(22 s+15) / 6$. Then we have

$$
\|I u(T)\|_{L^{2}}^{2} \exp (2 \gamma T) \leq C_{4}\left(\left\|I u_{0}\right\|_{L^{2}}^{2}+\gamma^{-2}\|I f\|_{L^{2}}^{2} \exp (2 \gamma T)\right) .
$$

\section{Proof of Theorem 1.2}

In this section, we prove Theorem 1.2.

Proof of Theorem 1.2. Let $0<\varepsilon<(22 s+15) / 6$. Since $-22 s / 3(5-2 \varepsilon)<1$, we can choose $T_{1}>0$ large enough such that

$$
\begin{aligned}
\exp \left(2 \gamma T_{1}\right) \geq & \left\|u_{0}\right\|_{H^{s}}^{2}\|f\|_{L^{2}}^{-2} \\
& \times \max \left\{\gamma^{\frac{-22 s}{3(5-2 \varepsilon)}},\left(C_{2} T_{1}\right)^{\frac{-2 s}{\varepsilon-}},\left(2 C C_{3}^{-1}\left\|u_{0}\right\|_{H^{s}}^{2}\right)^{\frac{-22 s}{15+22 s-6 \varepsilon}}\right. \\
& \left.\left(2 C_{3}^{-1} \gamma^{-2}\|f\|_{L^{2}}^{2} \exp \left(2 \gamma T_{1}\right)\right)^{\frac{-22 s}{3(5-2 \varepsilon)}}\right\}
\end{aligned}
$$

where $C_{2}$ and $C_{3}$ are the same as in Proposition 3.8 and $C$ as in (2.4), and $T_{1}$ depends only on $\|f\|_{L^{2}}, \gamma$ and $\left\|u_{0}\right\|_{H^{s}}$. Set

$$
\begin{gathered}
N=\max \left\{\gamma^{\frac{11}{3(5-2 \varepsilon)}},\left(C_{2} T_{1}\right)^{\frac{1}{\varepsilon-}},\left(2 C C_{3}^{-1}\left\|u_{0}\right\|_{H^{s}}^{2}\right)^{\frac{11}{15+22 s-6 \varepsilon}},\right. \\
\left.\left(2 C_{3}^{-1} \gamma^{-2}\|f\|_{L^{2}}^{2} \exp \left(2 \gamma T_{1}\right)\right)^{\frac{11}{3(5-2 \varepsilon)}}\right\} .
\end{gathered}
$$

Then we have

$$
\begin{aligned}
& N^{\frac{3(5-2 \varepsilon)}{11}} \geq \gamma, \quad N^{\varepsilon-} \geq C_{2} T_{1}, \\
& \left\|I u_{0}\right\|_{L^{2}}^{2} \leq C N^{-2 s}\left\|u_{0}\right\|_{H^{s}}^{2} \leq \frac{1}{2} C_{3} N^{\frac{3(5-2 \varepsilon)}{11}}, \\
& \gamma^{-2}\|f\|_{L^{2}}^{2} \exp \left(2 \gamma T_{1}\right) \leq \frac{1}{2} C_{3} N^{\frac{3(5-2 \varepsilon)}{11}} .
\end{aligned}
$$

Therefore, from Proposition 3.8, we obtain

$$
\left\|I u\left(T_{1}\right)\right\|_{L^{2}}^{2} \leq C_{4}\left(C N^{-2 s}\left\|u_{0}\right\|_{H^{s}}^{2} \exp \left(-2 \gamma T_{1}\right)+\gamma^{-2}\|f\|_{L^{2}}^{2}\right) .
$$

From (4.1) and (4.2), we have

$$
N^{-2 s}\left\|u_{0}\right\|_{H^{s}}^{2} \exp \left(-2 \gamma T_{1}\right) \leq\|f\|_{L^{2}}^{2},
$$

and thus

$$
\left\|u\left(T_{1}\right)\right\|_{H^{s}}^{2} \leq\left\|I u\left(T_{1}\right)\right\|_{L^{2}}^{2} \leq C_{4}\left(C+\gamma^{-2}\right)\|f\|_{L^{2}}^{2} \equiv K_{1},
$$

where $K_{1}$ depends only on $\|f\|_{L^{2}}$ and $\gamma$. 
We fix $T_{2}>0$ and solve Eq. (1.1) on $\left[T_{1}, T_{1}+T_{2}\right]$ with initial data $u\left(T_{1}\right)$. Let $K_{2}>0$ be large enough such that for arbitrary $t>0$, it holds that

$$
\begin{gathered}
K_{2} \exp (2 \gamma t) \geq \max \left\{\gamma^{\frac{-22 s}{3(5-2 \varepsilon)}},\left(C_{2} t\right)^{\frac{-2 s}{\varepsilon-}},\left(2 C C_{3}^{-1} K_{1}\right)^{\frac{-22 s}{15+22 s-6 \varepsilon}},\right. \\
\left.\left(2 C_{3}^{-1} \gamma^{-2}\|f\|_{L^{2}} \exp (2 \gamma t)\right)^{\frac{-22 s}{3(5-2 \varepsilon)}}\right\},
\end{gathered}
$$

where $K_{2}$ depends only on $\|f\|_{L^{2}}$ and $\gamma$. Put $N^{-2 s}=K_{2} \exp \left(2 \gamma T_{2}\right)$, then by using Proposition 3.8 again, we obtain

$$
\begin{aligned}
\left\|I u\left(T_{1}+T_{2}\right)\right\|_{L^{2}}^{2} & \leq C_{4}\left(\left\|I u\left(T_{1}\right)\right\|_{L^{2}}^{2} \exp \left(-2 \gamma T_{2}\right)+\gamma^{-2}\|f\|_{L^{2}}^{2}\right) \\
& \leq C_{4}\left(K_{1} \exp \left(-2 \gamma T_{2}\right)+\gamma^{-2}\|f\|_{L^{2}}^{2}\right) \\
& \leq C_{4}\left(K_{1}+\gamma^{-2}\|f\|_{L^{2}}^{2}\right) \equiv K_{3},
\end{aligned}
$$

where $K_{3}$ depends only on $\|f\|_{L^{2}}$ and $\gamma$.

For $t>T_{1}$, set $T_{2}=t-T_{1}$. Define maps $M_{1}(t)$ and $M_{2}(t)$ by

$$
M_{1}(t) u_{0}=P_{2 N} S(t) u_{0}, \quad M_{2}(t) u_{0}=P^{2 N} S(t) u_{0},
$$

where $S(t) u_{0}=u(t)$ and $N=\left(K_{2} \exp \left(\gamma\left(t-T_{1}\right)\right)\right)^{1 /(-2 s)}$. Then for $t>T_{1}$, we have

$$
\begin{aligned}
& \left\|M_{1}(t) u_{0}\right\|_{L^{2}}^{2} \leq 2^{-2 s}\|I u(t)\|_{L^{2}}^{2} \leq 2 K_{3} \\
& \left\|M_{2}(t) u_{0}\right\|_{H^{s}}^{2} \leq N^{2 s}\|I u(t)\|_{L^{2}}^{2} \leq K_{2}^{-1} K_{3} \exp \left(-2 \gamma\left(t-T_{1}\right)\right) .
\end{aligned}
$$

Let $K=\max \left\{\left(2 K_{3}\right)^{1 / 2}, K_{2}^{-1 / 2} K_{3}^{1 / 2}\right\}$, and then we have (1.3) and (1.4). This completes the proof of Theorem 1.2 .

\section{References}

[1] Korteweg, D.J., de Vries, G.: On the change of form of long waves advancing in a rectangular channel, and a new type of long stationary waves. Philos. Mag. 39, 422-443 (1895)

[2] Bourgain, J.: Fourier transform restriction phenomena for certain lattice subsets and applications to nonlinear evolution equations, II: the KdV-equation. Geom. Funct. Anal. 3, 209-262 (1993)

[3] Kenig, C., Ponce, G., Vega, L.: The Cauchy problem for the Korteweg-de Vries equation in Sobolev spaces of negative indices. Duke Math. J. 71, 1-21 (1993)

[4] Kenig, C., Ponce, G., Vega, L.: A bilinear estimate with applications to the KdV equation. J. Am. Math. Soc. 9, 573-603 (1996)

[5] Christ, M., Colliander, J., Tao, T.: Asymptotics, frequency modulation, and low regularity ill-posedness for canonical defocusing equations. Am. J. Math. 125, 1235-1293 (2003)

[6] Kenig, C., Ponce, G., Vega, L.: On the ill-posedness of some canonical dispersive equations. Duke Math. J. 106, 617-633 (2001) 
[7] Colliander, J., Keel, M., Stafillani, G., Takaoka, H., Tao, T.: Global well-posedness for KdV in Sobolev spaces of negative index. Electron. J. Differ. Equ. 26, 1-7 (2001)

[8] Colliander, J., Keel, M., Stafillani, G., Takaoka, H., Tao, T.: Sharp global wellposedness for $\mathrm{KdV}$ and modified $\mathrm{KdV}$ on $\mathbf{R}$ and T. J. Am. Math. Soc. 16, 705-749 (2003)

[9] Guo, Z.H.: Global well-posedness of Korteweg-de Vries equation in $H^{-3 / 4}(\mathbf{R})$. J. Math. Pures Appl. 91, 583-597 (2009)

[10] Kappeler, T., Topalov, P.: Global wellposedness of $\mathrm{KdV}$ in $H^{-1}(\mathbf{T}, \mathbf{R})$. Duke Math. J. 135, 327-360 (2006)

[11] Kappeler, T., Pöschel, J.: On the periodic KdV equation in weighted Sobolev spaces. Ann. I. H. Poincaré-AN 26, 841-853 (2009)

[12] Ghidaglia, J.: Weakly damped forced Korteweg-de Vries equations behave as a fnite-dimensional dynamical system in the long time. J. Differ. Equ. 74, 369-390 (1988)

[13] Ghidaglia, J.: A note on the strong convergence towards attractors of damped forced KdV equations. J. Differ. Equ. 110, 356-359 (1994)

[14] Moise, I., Rosa, R.: On the regularity of the global attractor of a weakly damped, forced Korteweg-de Vries equation. Adv. Differ. Equ. 2, 257-296 (1997)

[15] Goubet, O.: Asymptotic smoothing effect for weakly damped forced KdV equations. Disc. Contin. Dyn. Syst. 6, 625-644 (2000)

[16] Goubet, O., Rosa, R.: Asymptotic smoothing and the global attractor of a weakly damped KdV equation on the real line. J. Differ. Equ. 185, 25-53 (2002)

[17] Tsugawa, K.: Existence of the global attractor for weakly damped, forced KdV equation on Sobolev spaces of negative index. Comm. Pure Appl. Anal. 3, 301-318 (2004)

[18] Temam, R.: Infinite dimensional dynamical systems in mechanics and physics, 2nd edn. Appl. Math. Sci. Vol. 68, Springer, New York (1997)

X. Yang

School of Business Administration,

South China University of Technology,

Guangzhou, 510640 Guangdong,

People's Republic of China

e-mail: xyyangmath@gmail.com

Received: 06 June 2010.

Accepted: 20 December 2010. 